\title{
Desarrollo matemático de la frontera eficiente de portafolio y diversificación de cultivos hortícolas en México
}

Antonio Kido-Cruz*y MA. Teresa Kido-CruZ**

\section{RESUMEN}

La solución de problemas de optimización cuadrática suele ser una tarea difícil y cuando se utilizan series históricas sobre rendimientos agrícolas es poco frecuente que los porcentajes asignados sean no-negativos. En finanzas, la explicación de este evento se resuelve con las ventas en corto; sin embargo, para otros tipos de activos reales como la generación de electricidad o la agricultura, la explicación no es del todo satisfactoria. Este artículo estima el potencial de diversificación hortícola en México del modelo matemático de Markowitz. Los resultados muestran que usando todos los cultivos seleccionados no es posible encontrar porcentajes positivos, por lo que la interpretación de los resultados resulta inapropiada en este contexto. Cuando la cantidad de cultivos se restringe en función de una matriz de correlación débil entre las rentabilidades de los cultivos seleccionados, se encuentra una región donde los pesos de todos los cultivos son positivos, pero teoréticamente resulta insuficiente.

Palabras clave: cultivos hortícolas, teoría óptima, frontera eficiente. Clasificación JEL: C; C6; C65.

\footnotetext{
*Facultad de Contaduría y Ciencias Administrativas, Universidad Michoacana de San Nicolás de Hidalgo, Michoacán, México. Correo-e: akido42@hotmail.com.

** Universidad del Papaloapan, Oaxaca, México. Correo-e: terekido@hotmail.com.
} 


\section{ABSTRACT}

Mathematical development of the efficient portfolio frontier and diversification of agricultural crops in Mexico

The resolution of problems of optimization of the efficient frontier is usually a difficult task and when using historical series on crop yields it is rare that the percentages assigned are non-negative. In finance, the explanation of this event is resolved with short sales, however, for other types of real assets such as those related to energy or agriculture the explanation is not entirely satisfactory. The main objective of this research is to estimate the potential for horticultural diversification in Mexico using a Markowitz's mathematical model. The results show that, using only the optimal portfolio theory, it is not possible to find positive percentages for all the crops incorporated in the study. When the quantity of crops is restricted based on a weak correlation matrix among selected crop yieldst, it is possible to find a region where the weights of all the crops are positive but results are theoretically incomplete.

Keywords: electricity generation, optimal theory, efficient frontier. JEL Classification: C; C6; C65.

\section{INTRODUCCIÓN}

Es posible justificar que, en un gran número de países, los sistemas agrícolas actuales han logrado proporcionar una cantidad suficiente de alimentos para una demanda creciente. También es pertinente señalar que la variación en un número importante de factores que inciden en las actividades agropecuarias, como las condiciones climáticas, la creciente demanda social de una agricultura de bajos insumos y la corresponsabilidad ambiental generan que la agricultura sea cada vez más sensible al riesgo y la incertidumbre (OCDE, 2011). A esta variabilidad es importante agregar que la volatilidad de precios ha creado, desde siempre, un nivel adicional de riesgo en los ingresos de los agricultores (Kimura et al., 2010). Estos problemas son particularmente severos en los sistemas agrícolas industriales que principalmente dependen del monocultivo y de un alto nivel de insumos sintéticos (Paut et al., 2019).

En el caso particular de México, debemos agregar que el patrón de cultivos ha evolucionado por las características propias de la actividad y las condiciones climatológicas que determinan en gran medida 
los volúmenes anuales de producción, afectados directamente por las variaciones en la superficie sembrada y cosechada, así como los rendimientos unitarios. En épocas más recientes, la producción agropecuaria ha respondido a la demanda derivada de la apertura comercial del país, acentuada con la firma del Tratado de Libre Comercio de América del Norte en 1994 (Cruz-Delgado et al., 2013). Uno de los aspectos positivos del potencial nuevo tratado comercial entre México, Canadá y Estados Unidos (T-MEC) es el hecho de que no presenta cambios importantes en el comercio agrícola.

Las exportaciones agrícolas de Estados Unidos a México tienen un valor aproximado de 19 mil millones de dólares anuales e incluyen productos como trigo, lúpulo, maíz, soya y otros granos, carne de vaca, partes de pollo y cerdo. Las importaciones agrícolas desde México hacia Estados Unidos totalizaron 25 mil millones de dólares en 2017; incluyeron verduras y frutas frescas, pero también vino y cerveza, alimentos procesados, y frutas y verduras procesadas. Por lo tanto, parece claro que México seguirá importando productos agrícolas con un alto nivel de mecanización y procesamiento, mientras que las exportaciones de México seguirán estando representadas, principalmente, por frutas y verduras que requieren mucha mano de obra (Gantz, 2019). El objetivo de este trabajo de investigación es estimar la relación riesgorendimiento para la determinación de la frontera eficiente para cultivos hortofrutícolas en México. Es importante destacar que el análisis considera el supuesto de que existe la posibilidad, en términos de la actividad hortofrutícola, de ser reasignada en forma flexible.

El siguiente artículo considera las siguientes secciones: en el primer apartado aparece la introducción; en el apartado dos se presenta una breve revisión de literatura; la sección tres desarrolla los materiales y métodos; en la sección cuatro se describen las fuentes de información, y finalmente los resultados son presentados en la sección cinco.

\section{ESTADO DEL ARTE}

La diversificación en la agricultura puede significar cualquiera de las tres situaciones: (i) un cambio de las actividades agrícolas a las no agrícolas, (ii) un cambio de un cultivo (o empresa) menos rentable a un cultivo (o empresa) más rentable, (iii) uso de recursos en actividades diversas pero complementarias (Vyas, 1996). La justificación para la diversifica- 
ción de cultivos agrícolas proviene de las oportunidades que ofrece para reducir los riesgos de producción y precio (Deshpande et al., 2007). La teoría moderna de portafolio se desarrolló en el contexto de los activos financieros, pero el concepto puede aplicarse a una amplia variedad de entornos bajo incertidumbre y toma de decisiones asociadas con el riesgo, incluso en el caso de la selección de cultivos (Barkley et al., 2010).

Para el caso de México, los trabajos de Avilés-Cano et al. (2006), Díaz-Carreño et al. (2007), De Jesús-Uribe et al., (2009) y León-Herrera et al. (2015) utilizan el método propuesto por Markowitz en la elección de un portafolio de inversión para productos agrícolas, hortícolas y/o pecuarios. En este sentido, podemos referir los siguientes trabajos aplicados a otros activos reales, como el caso de la generación de electricidad por tipo de tecnología (Gómez-Rios et al., 2018; Awerbuch y Spencer, 2006); a bienes raíces (Benjamin et al., 2001; Buetov y Jhonson, 2001); a pensiones (Brinson et al., 1986).

\section{MATERIALES Y MÉTODOS}

Dado que la incertidumbre implica, implícitamente, un riesgo para evaluar la incertidumbre, debemos tener una medida del riesgo. Al medir el riesgo, es importante evaluar la variabilidad de un evento incierto en relación con su valor esperado. El coeficiente de variación, que es la relación entre la desviación estándar y la media, proporciona una unidad conveniente para medir la relación entre la variabilidad de los rendimientos y el rendimiento esperado. El cálculo del coeficiente de variación para diferentes cultivos indica al agricultor la cantidad relativa de incertidumbre asociada con cada cultivo (Mukherjee, 2010).

\subsection{Conjunto de cartera eficiente cuando todos los valores corren riesgo}

En el formalismo de la teoría de optimización de portafolios de Markowitz (1959) tenemos como ingredientes principales un conjunto de $m$ activos. De cada uno de ellos se desprende una serie en el tiempo de $T$ datos (medido en unidades de inversión); en el caso de portafolios de inversión, corresponden a los precios de cierre de los activos, de donde obtenemos $T-1$ retornos, siguiendo la ecuación 


$$
r_{t}=\left(P_{t}-P_{t-1}\right) / P_{t-1} \quad(\text { adimensional })
$$

Estos T-1 retornos forman una serie en el tiempo que tiene ese mismo número de elementos.

\section{Retorno Esperado (Media del Activo j/Retorno Esperado de cada Activo j):}

Para cada uno de los $\mathrm{m}$ activos podemos calcular su media (o promedio) haciendo uso de la siguiente fórmula

$$
\mu_{j}=\frac{1}{T-1} r_{i}, j=1, \ldots, m
$$

\subsection{Desviación estándar (riesgo de cada activo j):}

Es posible calcular una medida de dispersión alrededor de media de cada activo j haciendo uso de la ecuación de la desviación estándar:

$$
\sigma_{j}=\sqrt{\frac{1}{T-1} \sum_{i=1}^{T-1}\left(r_{i}-\mu_{j}\right)^{2}, j}=1, \ldots, m
$$

Para $m$ activos tenemos que el valor del riesgo para cada uno de los activos está dado. Sin embargo, Markowitz (1959) resuelve esta limitante con un procedimiento para la diversificación: calcula una combinación lineal de ellos bajo el supuesto de que se tiene un presupuesto inicial $\mathrm{x}_{\mathrm{o}}$ (unidades de inversión) para distribuir de forma inteligente a manera de inversión entre ellos y se pregunta ¿cuál podría ser una forma más eficiente de invertir $\mathrm{x}_{\mathrm{o}}$ unidades de forma diferenciada entre los $\mathrm{m}$ activos? Lo primero que debe hacer es respetar la restricción dada por el

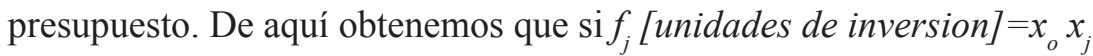
[adimensional], donde $x_{o}=\sum_{i=f}^{m} f_{j}=x_{o} \sum_{i=I}^{m} x_{j}$. Así, tenemos que al dividir entre $x_{o}$ a la izquierda y a la derecha obtenemos:

$$
1=\sum_{i=1}^{m} x_{j}
$$


Nótese que $\mathrm{x}_{\mathrm{j}}$ es adimensional, así que solamente representa el porcentaje asignado a cada activo en una inversión $\left(0<\mathrm{x}_{\mathrm{j}}<1\right.$, para todo $j=1, \ldots, m)$.

\subsection{Portafolio de inversión con $m$ activos}

Si tenemos $m$ activos (con riesgo) podemos definir un portafolio de inversión mediante un conjunto ordenado de porcentajes $\left(x_{p}, \ldots, x_{m}\right)$ $p=: x_{p}$. Cada variación de los valores de cada componente $\mathrm{x}_{\mathrm{j}}$ da como resultado un portafolio diferente.

En el contexto original de la teoría de portafolios existe una explicación posible y razonable para $x_{i}<0$, por lo que se podía relajar esta restricción suponiendo que existe la venta en corto; sin embargo, no es usual encontrar explicaciones convincentes en este tipo de transacción en el ámbito del sector agropecuario.

\subsection{Retorno esperado de un portafolio de inversión}

Dado un portafolio de inversión, el valor esperado del retorno, en función de $x_{p}$, se puede definir como:

$$
E\left(x_{p}\right)=\frac{1}{m} \sum_{i=1}^{m} x_{i} \mu_{i}
$$

Obsérvese que i es un índice que recorre todos los posibles activos, $x_{i}$ son los porcentajes asignados a cada uno de los m activos, $\mu_{i}$ son las medias de cada uno de los activos que han sido calculados con antelación.

\subsection{Riesgo de un portafolio de inversión}

El riesgo del portafolio queda definido por la varianza, que se puede escribir como:

$$
\sigma\left(\underline{x}_{p}\right)=\sqrt{\left(\sum_{i=1}^{m} \sum_{j=1}^{m} x_{i} x_{j} \sigma_{i j}\right)},
$$

Donde $\sigma_{i j}$ son las componentes de la matriz de varianza-covarianza, la cual es una matriz cuadrada de $m \times m$ dimensiones, que en su diagonal 
está constituida por un vector de $\mathrm{m}$ dimensiones $\left(\sigma_{11}, \sigma_{22}, \sigma_{33}, \ldots, \sigma_{m m}\right)$, cuyos elementos son la varianza (desviación estándar al cuadrado de cada activo). El elemento fuera de la diagonal $\sigma_{i j}$ es la covarianza entre los activos i y $\mathrm{j}$, que viene dado por:

$$
\sigma_{i j}=\frac{1}{T-1} \sum_{l=1}^{T-1}\left(r_{l}-\mu_{i}\right)\left(r_{l}-\mu_{j}\right)
$$

Donde $l$ es un índice de suma que recorre todos los T-1 datos históricos, y los índices i y j se refieren a los activos en cuestión.

\subsection{La función objetivo}

Para un conjunto de $m$ activos y una combinación lineal de éstos dada por los componentes del vector de pesos $x_{p}$, es posible definir una función que devuelve un escalar:

$$
\begin{gathered}
F:\left(\underline{x}_{p}\right) \rightarrow R \\
\underline{x}_{p} \rightarrow F\left(\underline{x}_{p}\right):=\frac{1}{2} \sigma^{2}\left(\underline{x}_{p}\right),
\end{gathered}
$$

Donde $F$ es la función objetivo definida como la mitad de la desviación estándar elevada al cuadrado. Cada valor de una n-tupla de pesos de un portafolio, $F\left(x_{p}\right)$ da, por lo general, un valor diferente.

\subsection{Problema de optimización de portafolios}

El acierto de Markowitz fue relacionar la desviación estándar con el riesgo, la cual tiene la tarea de asignar una medida cuantitativa. El problema de reducir el riesgo de un portafolio, dado un valor de retorno esperado, se puede escribir de forma matemática como un problema de optimización con restricciones m-dimensional, que viene dado por:

$$
\begin{gathered}
\min _{\underline{x}_{p}} F\left(\underline{x}_{p}\right), \\
\text { s.a. } E\left(\underline{x}_{p}\right)=E_{p}, \\
\sum_{i=1}^{m} x_{i}=1 .
\end{gathered}
$$

La solución a este problema es $x_{p}{ }^{*}$, tal que cumple con ser el mínimo de todos los valores de la función objetivo Fy además con las restricciones que $E\left(x_{p}{ }^{*}\right)=E_{p} y \sum_{i=r}^{m} x_{i}^{*}=1$. 
Podemos cambiar el problema de optimización con restricciones $m$-dimensional por un problema sin restricciones $m+2$-dimensional haciendo uso de los multiplicadores de Lagrange:

$$
\min _{\left(\underline{x}_{p}, \lambda_{1}, \lambda_{2}\right)} L:=F\left(\underline{x}_{p}\right)-\gamma_{1} g_{1}\left(\underline{x}_{p}\right)-\gamma_{2} g_{2}\left(\underline{x}_{p}\right)
$$

Donde $g_{1}$ y $g_{2}$ se definen usando las restricciones del problema

$$
\begin{aligned}
& g_{1}\left(\underline{x}_{p}\right):=E\left(\underline{x}_{p}\right)-E_{p} \\
& g_{2}\left(\underline{x}_{p}\right):=\sum_{i=1}^{m}\left(\underline{x}_{p}\right)_{i}-1 .
\end{aligned}
$$

\subsection{Resolviendo el problema sin restricciones}

Las condiciones necesarias para encontrar un punto estacionario para el problema sin restricciones vienen dadas por:

$$
\nabla_{\left(\underline{x}, \lambda_{1}, \lambda_{2}\right)} L=0
$$

El operador $\nabla\left(\underline{x}, \lambda_{1}, \lambda_{2}\right)$ significa que realizaremos $m+2$ derivadas parciales de $L$; las acomodaremos como los componentes de un vector columna y las igualamos a un vector columna con ceros como componentes:

\begin{tabular}{|c|c|c|c|c|}
\hline$\frac{\partial}{\partial x_{1}} L=0$, & $\ldots$ & $\frac{\partial}{\partial x_{m}} L=0$ & $\frac{\partial}{\partial \lambda_{1}} L=0$ & $\frac{\partial}{\partial \lambda_{2}} L=0$ \\
\hline
\end{tabular}

Para encontrar el punto estacionario $\left(\underline{x}_{p}^{*}, \lambda_{1}^{*}, \lambda^{*}\right)$ es necesario resolver el sistema de ecuaciones algebraicas resultante de la solución de las derivadas parciales anteriores, que se puede escribir de forma explícita de la siguiente forma:

$$
0=\sum_{j=1}^{m} x_{j} \sigma_{i j}-\gamma_{1} E_{i}-\gamma_{2} \cdot 1, i=1, \ldots m,
$$




$$
\begin{aligned}
& 0=E-\sum_{1}^{m} x_{i} E_{i} \\
& 0=1-\sum_{1}^{m} x_{i}
\end{aligned}
$$

Nótese que hemos obviado las barras y los asteriscos de nuestra notación anterior, de tal manera que $\left(\underline{x}_{p}^{*}\right)_{j}=x_{j}, \gamma_{1}^{*}=\gamma_{1}$ y $\gamma_{2}^{*}=\gamma_{2}$.

Aquí podemos observar que hay $\mathrm{m}+2$ ecuaciones con $m+2$ incógnitas en lugar de un sistema de ecuaciones diferenciales parciales. Por comodidad pasaremos a la forma matricial del problema:

$$
\begin{gathered}
\left(\sigma \underline{x}^{T}\right)_{i}-\left(\gamma_{1} \underline{E}\right)_{i}-\left(\gamma_{2} \underline{1}\right)_{i}=0, i=1, \ldots, m, \\
0=E-\underline{x}^{T} \underline{E} \\
0=1-\underline{1}^{T} \underline{x}
\end{gathered}
$$

El vector de pesos (columna) $\underline{x}=\left(x_{1}, x_{2}, \ldots, x_{m}\right)^{T}$ es el que contiene los porcentajes (que serán parte principal de la solución del problema de optimización junto con los multiplicadores de Lagrange $\lambda_{1} y \lambda_{2}$ ). Especificamos también que $\underline{x}^{T}=\left(x_{1}, x_{2}, \ldots, x_{m}\right)$ es un vector fila o renglón de $\mathrm{m}$ entradas con los mismas componentes que $\underline{x}$. Aquí $\underline{E}=\left(\mu_{1}, \ldots, \mu_{m}\right)^{\mathrm{T}}$ es el vector columna que contiene los promedios o medias de los datos históricos. Por otra parte, $\underline{l}=(1, \ldots, 1)^{T}$ es el vector que columna de $m$ componentes, que tiene solamente entradas con valor 1 .

Podemos destacar algunas operaciones matriciales que aparecen en el sistema (3'):

El componente de un vector se destaca con un subíndice, $j$ por ejemplo, donde en la ecuación (3a') aparece en tres ocasiones señalando que es el componente $j$-ésima de una entidad vectorial.

El producto de un vector fila $v^{T}=\left(v_{1}, \ldots, v_{m}\right)^{T}$ por un vector columna $\underline{w}^{T}=\left(w_{l}, \ldots, w_{m}\right)$, como en el caso de (3b') y (3c'), representa el producto escalar entre dos vectores se define como 


$$
\underline{v}^{T} \underline{w}=\sum_{l=1}^{m} v_{l} w_{l} .
$$

Haremos uso de la notación matricial para encontrar la solución al sistema (3'). Comenzamos con la ecuación (3a')

$$
\sigma \underline{x}-\gamma_{1} \underline{E}-\gamma_{2} \underline{1}=\underline{0}
$$

Donde $\underline{Q}^{T}=(0, \ldots, 0)^{T}$ es un vector columna con ceros en todas sus entradas (componentes). Definimos la matriz inversa de $\sigma$ como aquella que tiene los componentes $v_{i j}$

$$
\left[\sigma^{-1}\right]_{i j}:=v_{i j}
$$

Analíticamente hablando (es decir, fuera del ámbito de los ordenadores) para que exista la matriz inversa de $\sigma$, la condición suficiente que se tienen que cumplir es que $|\sigma|=\operatorname{det}(\sigma) \neq 0$. Por tanto, al multiplicar la primera ecuación por la matriz inversa tenemos:

$$
\sigma^{-1} \sigma \underline{x}-\gamma_{1} \sigma^{-1} \underline{E}-\gamma_{2} \sigma^{-1} \underline{1}=\sigma^{-1} \underline{0},
$$

Nótese que $\sigma^{(-1)} \sigma=1_{(m \times m)}$, donde $1_{(m \times m)}$ es la matriz identidad que tiene unos en la diagonal y ceros en todas las demás entradas. Al multiplicar la matriz inversa por un vector obtenemos otro vector de la misma dimensión que el inicial. Por último, observamos la sencilla multiplicación $\sigma^{(-1)} 0=0$ del lado derecho de la ecuación.

Finalmente, el resultado de la ecuación anterior es:

$$
\underline{x}-\gamma_{1} \sigma^{-1} \underline{E}-\gamma_{2} \sigma^{-1} \underline{1}=\underline{0},
$$

Obsérvese que si $\gamma_{1}$ y $\gamma_{2}$ fueran conocidos, inmediatamente tendríamos los valores solución, ya que el vector $E$ y la matriz $\sigma^{(-1)}$ son conocidos o calculables de los datos del problema. Por tanto, nuestra tarea de aquí en adelante es encontrar $\gamma_{1}$ y $\gamma_{2}$.

Podemos escribir para la componente k-ésima de la ecuación (4) como 


$$
x_{k}=\gamma_{1} \sum_{j=1}^{m} v_{k j} E_{j}+\gamma_{2} \sum_{j=1}^{m} v_{k j}, k=1, \ldots m .
$$

Al multiplicar la ecuación anterior por $E_{k}$ y sumar sobre $k$ tenemos:

$$
\sum_{1}^{m} x_{k} E_{k}=\gamma_{1} \sum_{1}^{m} \sum_{1}^{m} v_{k j} E_{j} E_{k}+\gamma_{2} \sum_{1}^{m} \sum_{1}^{m} v_{k j} E_{k},
$$

En notación matricial se obtiene:

$$
\underline{x}^{T} \underline{E}=\gamma_{1} \underline{E}^{T} \sigma^{-1} \underline{E}+\gamma_{2} \underline{1}^{T} \sigma^{-1} \underline{E}
$$

En el lado derecho de esta ecuación usaremos la definición del valor esperado del retorno de un portafolio en su forma matricial, $x^{T} \underline{E}=: \underline{E}_{p}$, donde $E_{p}$ es un dato del problema, así:

$$
E_{p}=\gamma_{1} A+\gamma_{2} B
$$

Donde se han definido dos de los tres escalares de Merton (1972: 1853):

$$
A:=\underline{E}^{T} \sigma^{-1} \underline{E} ; B:=\underline{1}^{T} \sigma^{-1} \underline{E},
$$

Que se pueden calcular a partir de la matriz inversa de varianza-covarianza y del vector de medias; por lo tanto, son valores que se pueden considerar datos del problema. Por otra parte, si hacemos la operación de contracción, que es la suma de todas las componentes del vector $x_{k}$, obtenemos:

$$
\sum_{1}^{m} x_{k}=\gamma_{1} \sum_{1}^{m} \sum_{1}^{m} v_{k j} E_{j}+\gamma_{2} \sum_{1}^{m} \sum_{1}^{m} v_{k j},
$$

Esto también tiene su forma matricial, representada por:

$$
\underline{1}^{T} \underline{x}=\gamma_{1} B+\gamma_{2} C .
$$


Donde podemos ver que el tercer escalar obtenido de Merton (1972) es:

$$
C \equiv \sum_{1}^{m} \sum_{1}^{m} v_{k j}
$$

El lado izquierdo de esta ecuación es igual a 1 por ser la restricción presupuestaria a la que está sujeto el problema y, por lo tanto:

$$
1=\gamma_{1} B+\gamma_{2} C
$$

Así, (6) y (9) forman un sistema de dos ecuaciones con dos incógnitas $\left(\gamma_{1}, \gamma_{2}\right)$, que tiene como solución:

$$
\gamma_{1}=\frac{(E C-B)}{D}, \gamma_{2}=\frac{(A-E B)}{D}
$$

Esta solución se puede ver de forma inmediata si usamos la fórmula de Kramer y si, además, para que exista dicha solución pedimos que el discriminante $B C-A^{2}=: D \neq 0$.

Una vez obtenido $\left(\gamma_{1}, \gamma_{2}\right)$, sustituimos en (5) o en su forma desarrollada para obtener la solución para los porcentajes de un portafolio eficiente:

$$
x_{k}=\frac{E \sum_{1}^{m} v_{k j}\left(C E_{j}-A\right)+\sum_{1}^{m} v_{k j}\left(B-A E_{j}\right)}{D}, k=1, \ldots, m .
$$

\subsection{Frontera eficiente}

De forma gráfica, la frontera eficiente está descrita por todos aquellos puntos en el plano $\sigma-E$ en los que para un cierto valor dado de $E_{p}$ encontramos el $\sigma_{p}$, mínimo que una combinación lineal con pesos $x_{p}$ puede dar.

Para obtener el lugar geométrico en $R^{2}$ con el eje de las abscisas igual al riesgo $\sigma$ y el eje de las ordenadas igual al retorno del portafolio $E p$, construimos una expresión que relacione estas dos cantidades al multiplicar (3a) por $x_{i}$ y sumar de $i=1, \ldots, m$ :

$$
\sum_{i=1}^{m} \sum_{j=1}^{m} x_{i} x_{j} \sigma_{i j}=\gamma_{1} \sum_{1}^{m} x_{i} E_{i}+\gamma_{2} \sum_{1}^{m} x_{i}
$$


El lado izquierdo de esta expresión es la definición de la varianza del portafolio, es decir, $\sigma^{2}\left(x_{p}\right)$, mientras que del lado derecho multiplicamos a $\gamma^{l}$ al valor esperado del retorno del portafolio $E_{p}$, y a $\gamma^{2}$ la ecuación de presupuestos que es 1 , obtenemos:

$$
\sigma^{2}\left(x_{p}\right)-\frac{1}{C}=\frac{C}{D}\left(E_{p}-\frac{A}{C}\right)^{2}
$$

Si C y D son mayores que cero, entonces el lado derecho de (12) siempre será positiva, ya que está escrito en términos de $\left(E_{p}-\frac{A}{c}\right)^{2} \geq 0$. Cuando $E_{p}=\frac{A}{c}$, este término será cero y por lo tanto $\sigma^{2}\left(x_{p}\right)=\frac{1}{c}$.

Definimos $\underline{E} \equiv \frac{A}{C}$ como el valor esperado mínimo de un portafolio que tiene la varianza mínima $\underline{\sigma}^{2} \equiv \frac{1}{c}$ (Merton, 1972: 1854).

Definimos $x_{k}$ para que sea la proporción del portafolio de varianza mínima invertida en el activo $k_{t h}$, entonces de (9),

$$
\underline{x}_{k}=\frac{\sum_{1}^{m} v_{k j}}{c}, k=1, \ldots, m
$$

Sin embargo, es habitual presentar la frontera en el plano de desviación estándar de la media en lugar del plano de la media-varianza, por lo que su representación geométrica quedaría como:

$$
\frac{\sigma^{2}}{\frac{1}{C}}-\frac{\left(E-\frac{A}{C}\right)^{2}}{\frac{D}{C}}=1
$$

Esta ecuación representa, según la geometría analítica, una hipérbola que cumple y confirma las condiciones de un portafolio eficiente con mínima varianza en $\underline{E} \equiv \frac{A}{C}$ y una mínima varianza de $\underline{\sigma}^{2} \equiv \frac{1}{c}$.

\subsection{Pesos no negativos}

De acuerdo con Best y Grauer (1992), tanto en el modelo de la teoría óptima como el modelo de precios de activos de capital (Sharpe, 1970; Lintner, 1965), los portafolios generados con porcentajes de inversión positivos tendrán la característica de ser eficientes dentro de la relación de media-varianza. Sin embargo, este tipo de portafolios (aquel en donde 
todos los pesos son positivos) no son tan comunes, sobre todo si se usan datos de series históricas de los activos. Sus resultados indican que, en el mejor de los escenarios, sólo es posible identificar un segmento de la frontera eficiente donde todos los pesos son no negativos.

Escrito de forma paramétrica, Markowitz se puede escribir de la siguiente forma:

$$
\max \left\{t E^{\prime} x-\frac{1}{2} x^{\prime} \sigma x \mid 1^{\prime} x=1\right\}
$$

Donde $\mathrm{t}$ es un parámetro escalar, $x$ es el n-vector de pesos de portafolio, y $l^{\prime} x=1$ es la restricción de presupuesto. La solución para este problema en términos del parámetro t para $x$ y $t$ es:

$$
\begin{gathered}
x(t)=\frac{\sigma^{-1} 1}{C}+t\left[\sigma^{-1}\left(E_{p}-\frac{1 A}{C}\right)\right] \\
\lambda(t)=-1 / C+t A / C
\end{gathered}
$$

Donde $A, B$ y $C$ son los escalares de Merton de la sección previa. Definamos ahora

$$
\begin{gathered}
h_{0}=\sigma^{-1} 1 / C \\
h_{1}=\sigma^{-1}\left(E_{p}-1 A / C\right)
\end{gathered}
$$

Con lo que se pueden expresar los pesos del portafolio como:

$$
x(t)=h_{o}+t h_{1}
$$

En esta ecuación se ve claramente, si observamos componente a componente,

$$
x_{i}(t)=\left(h_{o}\right)_{i}+t\left(h_{1}\right)_{i}, \text { con } i=1, \ldots, m,
$$


Que si $h l_{i}>0$, entonces $x_{i}(t)$ se incrementa conforme $t$ aumenta y será positivo dado que $t \geq(h 0) /(h 1)_{i}$.

Es posible determinar una cota máxima y una cota mínima para los valores de $t$, de tal forma que podamos garantizar la existencia de pesos positivos:

$$
\begin{aligned}
& t_{l}=\max \left\{-\left(h_{0}\right)_{i} /\left(h_{1}\right)_{i} \mid \text { para todo } i \text { con }\left(h_{1}\right)_{i}>0\right\} \\
& t_{u}=\min \left\{-\left(h_{0}\right)_{i} /\left(h_{1}\right)_{i} \mid \text { para todo } i \text { con }\left(h_{1}\right)_{i}<0\right\}
\end{aligned}
$$

De donde no es difícil deducir que

$$
\begin{aligned}
& x_{i}(t) \geq 0 \text { para todo } i \text { con }\left(h_{1}\right)_{i}>0 \text { y para todo } t \geq t_{l}, \\
& x_{i}(t) \geq 0 \text { para todo } i \text { con }\left(h_{1}\right)_{i}<0 \text { y para todo } t \leq t_{u} .
\end{aligned}
$$

Notemos que para que $\mathrm{x}(\mathrm{t})$ sea positivo en todas sus componentes para algún $\mathrm{t}$ se necesita que

$$
\left(h_{o}\right)_{i} \geq 0 \text { para todo } i \text { tal que }\left(h_{1}\right)_{i}=0 \text {. }
$$

Esto implica que $x(t)>0$ sólo y si $t_{l} \leq t \leq t_{u}$ y se satisface la restrcción de arriba (Best y Grauer, 1992: 519). De aquí es fácil obtener una fuerte conclusión que dice que si $t_{l}>t_{u}$, no existen componentes positivas de $x(t)$.

\section{INFORMACIÓN UTILIZADA}

Para este caso se analizó, en una primera etapa, la rentabilidad de 14 cultivos hortícolas: arroz palay, brócoli, calabacita, cebolla, chile verde, elote, frijol, maíz grano, papa, pepino, sorgo, grano, tomate rojo (jitomate), tomate verde y zanahoria, que de acuerdo con las estadísticas oficiales representan más de $95 \%$ del valor de la producción y alrededor de $90 \%$ de la superficie cosechada. El periodo de análisis abarcó del año 1980 a 2018 y la información se obtuvo del Sistema de Información Agroalimentaria de Consulta (Siacon, 2018). 


\section{RESULTADOS UTILIZANDO 14 CULTIVOS HORTíCOLAS}

\subsection{Resultados del desarrollo teórico del caso práctico patrón de cultivos hortícolas en México}

Es importante recordar que el criterio para encontrar todos los pesos positivos se cumple cuando $t_{l}>t_{u}$. En el caso del rango de la muestra analizada, el resultado fue que $t_{l}<t_{u}(1.3984$ no es menor que 0.61$)$, por lo que no es posible encontrar un portafolio eficiente que sólo tenga valores no negativos. Los resultados se presentan en el siguiente cuadro.

\section{CUADRO 1}

CRITERIO PARA ENCONTRAR PESOS POSITIVOS EN EL MOdELO CON CATORCE CUlTIVOS RIESGOSOS PARA OBTENER EL VALOR MAX (MÁXIMO) Y MIN (MÍNIMO) DE FORMA

CORRECTA

\begin{tabular}{ccccccc}
\hline ho & h1 & Xt=ho+t*h1 & ho/h1 & $(-)$ ho/h1 & Tlower & Tupper \\
\hline 0,0267 & $-0,043$ & 0,0180 & $-0,6175$ & 0,6175 & NA & 0,617 \\
\hline, 3367 & $-0,354$ & 0,265 & $-0,9507$ & 0,9507 & NA & 0,9507 \\
\hline 0,108 & $-0,088$ & 0,0912 & $-1,235$ & 1,235 & NA & 1,235 \\
\hline$-0,097$ & 0,109 & $-0,075$ & $-0,888$ & 0,888 & 0,888 & NA \\
\hline 0,0366 & $-0,046$ & 0,027 & $-0,796$ & 0,796 & NA & 0,796 \\
\hline 0,2849 & $-0,304$ & 0,224 & $-0,936$ & 0,936 & NA & 0,936 \\
\hline 0,0363 & $-0,013$ & 0,0336 & $-2,730$ & 2,730 & NA & 2,730 \\
\hline$-0,292$ & 0,2094 & $-0,251$ & $-1,398$ & 1,398 & 1,398 & NA \\
\hline 0,0287 & $-0,017$ & 0,0253 & $-1,675$ & 16,758 & NA & 1,675 \\
\hline 0,0541 & $-0,0405$ & 0,0459 & $-1,332$ & 1,332 & NA & 1,332 \\
\hline 0,2432 & $-0,179231$ & 0,2073 & $-1,357$ & 1,357 & NA & 1,357 \\
\hline 0,129 & $-0,114565$ & 0,106 & $-1,126$ & 1,126 & NA & 11,262 \\
\hline 0,0540 & $-0,0761$ & 0,0388 & $-0,7105$ & 0,7105 & NA & 0,7105 \\
\hline 0,05059 & $-0,0373$ & 0,0431 & $-1,356$ & 13,561 & NA & 1,356 \\
\hline & & & & & 1.397 & 0.617 \\
\hline
\end{tabular}

Fuente: elaboración propia.

Una vez convencidos de que con el simple hecho de usar la Teoría de Markowitz no es posible obtener todos los valores positivos, procedemos con el cálculo de la frontera eficiente, de donde obtenemos que el portafolio presenta el mínimo riesgo cuando $E=0.8 \%$ y el riesgo es de $\sigma=5.5 \%$. Asimismo, obtenemos que el vector x para este retorno y riesgo tiene los pesos: 
$x=(2.6 \% ; 33.6 \% ; 10.8 \% ;-9.7 \% ; 3.6 \% ; 28.4 \% ; 3.6 \% ;-29.2 \% ; 2.8 \%$;

$$
5.4 \% ; 2.4 \%, 12.9 \% ; 5.4 \%)
$$

Una interpretación bajo este contexto sería dejar de sembrar $29.2 \%$ maíz de grano y $9.7 \%$ de cebolla, lo que no suena del todo lógico. Otra alternativa es considerar sólo aquellos cultivos que presenten una correlación débil entre ellos, entendida como el hecho de que los elementos fuera de la diagonal de la matriz son pequeños en relación con los elementos diagonales de unos de la matriz (Best y Grauer, 1992). Cuando se impone esta restricción, los cultivos que sobreviven son: tomate rojo, papa, frijol, zanahoria y maíz grano. Sin embargo, esta solución debilita todo el embalaje teórico de la propuesta de frontera eficiente.

\section{CUADRO 2}

MATRIZ DE CORRELACIONES ENTRE CINCO CULTIVOS HORTÍCOLAS: TOMATE ROJO, PAPA, FRIJOL, ZANAHORIA Y MAÍZ GRANO

\begin{tabular}{cccccc}
\hline & $\begin{array}{c}\text { R Tomate rojo } \\
\text { (jitomate) }\end{array}$ & R Papa & R Frijol & Zanahoria & R Maíz grano \\
\hline R Tomate rojo (jitomate) & 1 & & & \\
R Papa & 0,02725 & 1 & & & \\
R Frijol & 0,10294 & $-0,36340$ & 1 & 1 & \\
\hline Zanahoria & $-0,20958$ & 0,30980 & $-0,05013$ & & \\
\hline R Maíz grano & 0,08828 & $-0,11529$ & 0,25579 & $-0,082843$ & 1 \\
\hline
\end{tabular}

Fuente: elaboración propia.

\subsection{Resultados para cinco cultivos agrícolas débilmente correlacionados}

El criterio para encontrar todos los pesos positivos se cumple cuando $t_{l}>t_{u}$. En el caso del rango de la muestra analizada, el resultado fue que $\mathrm{t}_{1}<\mathrm{t}_{\mathrm{u}}(0.0$ es menor que 0.101$)$, por lo que es posible encontrar un portafolio eficiente que sólo tenga valores positivos. Los resultados se presentan en el siguiente cuadro. 
CUADRO 3

CRITERIO PARA ENCONTRAR PESOS POSITIVOS EN EL MODELO CON CINCO CULTIVOS RIESGOSOS PARA OBTENER EL VALOR MAX (MÁXIMO) Y MIN (MÍNIMO) DE FORMA CORRECTA

\begin{tabular}{ccccccc}
\hline ho & h1 & Xt=ho+t*h1 & ho/h1 & $(-)$ ho/h1 & Tlower & Tupper \\
\hline 0,212 & $-1,702$ & $-0,127$ & $-0,125$ & 0,125 & NA & 0,125 \\
\hline, 205 & $-1,650$ & $-0,124$ & $-0,124$ & 0,124 & NA & 0,124 \\
\hline 0,156 & $-1,221$ & $-0,087$ & $-0,128$ & 0,128 & NA & 0,128 \\
\hline 0,155 & $-1,052$ & $-0,054$ & $-0,147$ & 0,147 & NA & 0,147 \\
\hline 0,269 & $-2,643$ & $-0,259$ & $-0,101$ & 0,101 & NA & 0,101 \\
\hline
\end{tabular}

Fuente: elaboración propia

Los resultados del Cuadro 3 indican que existe una región donde la tecnología libre de riesgo toma valores de la desviación estándar entre 0.02 y 0.09 , y los pesos de inversión son todos positivos. Para el resto del espectro existe cuando menos un valor negativo en la serie del vector $\mathrm{X}$. En la región de valores todos positivos para el vector $\mathrm{X}$ destacamos cuando se toma el valor de 0.03 . En este escenario, al cultivo de jitomate rojo le corresponde una participación de 16\%; papa, 16\%; frijol, 9.9\%; zanahoria, 3.3\%, y maíz, 56.7\% aproximadamente.

Este enfoque puede ser utilizado para arrojar nueva luz sobre el enfoque tradicional y métodos previamente propuestos. En particular, los resultados no concuerdan con los encontrados por Áviles et al. (2006), quienes determinan un portafolio óptimo conformado por ajo, brócoli, calabacita, chile verde, coliflor, frijol, garbanzo, lechuga, maíz, tomate y zanahoria. Cabe señalar que la afirmación de Estrada (2008) de que este método genera una cartera más eficiente no fue posible corroborarla en este análisis.

\section{CONCLUSIONES}

En el presente estudio se obtuvieron series históricas de los rendimientos para 14 cultivos hortícolas de mayor participación en superficie sembrada y cosechada en el país de 1980 a 2018. Tras aplicar programación cuadrática para encontrar el patrón de cultivos óptimos, se obtuvo que las participaciones de dos cultivos fueron negativas. El análisis se 
circunscribió a cinco cultivos bajo el supuesto de correlaciones débiles entre ellos. Se encontró una región donde todos los cultivos tienen participación positiva. La aplicación de la frontera eficiente a diferentes activos reales, como los de la agricultura, resulta compleja y es posible que muchos de los resultados encontrados en la revisión de literatura para México carezcan de una interpretación real en el contexto de la agricultura mexicana.

\section{REFERENCIAS}

Avilés-Cano, M., A. González-Estrada y M. Martínez-Damián (2006), “Análisis de riesgo, portafolios óptimos y diversificación en la agricultura", Agrociencia, 40(3), pp. 409-417. <http://www.colpos.mx/ agrocien/Bimestral/2006/may-jun/art-13.pdf>.

Awerbuch, S. y S. Yang (2006), "Portfolio-based electricity generation planning: policy implications for renewables and energy security", Mitigation and adaptation strategies for global change, 11 (3), pp. 693-710, <doi: 10.1007/s11027-006-4754-4>.

Barkley, A., H. Peterson y J. Shroyer (2010), "Wheat Variety Selection to Maximize Returns and Minimize Risk: An Application of Portfolio Theory", American Journal of Agricultural Economics (42), pp. 39-55.

Benjamin, J.D., G.S. Sirmans y E.N. Zietz (2001), "Returns and Risk on Real Estate and Other Investments: More Evidence", Journal of Real Estate Portfolio Management 7, pp. 183-214.

Best, M. y R. Grauer (1992), "Positively Weighted Minimum-Variance Portfolios and the Structure of Asset Expected Returns", Journal of Financial and Quantitative Analysis, 27(4), pp. 513-537. <doi:10.2307/2331138>.

Brinson, G.P., J.J. Diermeier y G.G. Schlarbaum (1986), “A Composite Portfolio Benchmark for Pension Plans", Financial Analysts Journal 42, pp. $15-24$.

Buetow, G.W. Jr. y R.R. Johnson (2001), "The Real Estate Asset Allocation Decision: Monetary Policy Implications", Journal of Real Estate Portfolio Management 7, pp. 215-223.

Cruz-Delgado, D., J. Leos-Rodríguez y R. Altamirano-Cárdenas (2013), “México: factores explicativos de la producción de frutas y hortalizas ante la apertura comercial", Revista Chapingo Serie Horticultura, 19 (3), pp. $267-$ 278.

De Jesús-Uribe L., M. Martínez-Damián y G. Ramírez-Valverde (2009), "Método de semivarianza y varianza para la selección de un portafolio óptimo", Revista Mexicana de Economía Agrícola y de los Recursos Naturales 2(1), pp. 103-113. <http://www.chapingo.mx/revistas/revistas/articulos/ doc/remecaren2840.pdf $>$.

Deshpande, R., P. Mehta y S. Khalil (2007), "Crop Diversification and Agricultural Labour in India", Indian Journal of Labour Economics, 50(4), pp. 597-610.

Díaz-Carreño, M., R. Juárez-Toledo y C. Gómez-Chagoya (2007), “Conformación de una cartera de inversión óptima de cultivos agrícolas para Mé- 
xico", Revista Economía, Sociedad y Territorio, 7(25), pp. 49-63. <http:// est.cmq.edu.mx/index.php/est/ article/view/231/237>.

Estrada, J. (2008), "Mean-semivariance optimization: A heuristic approach", Journal of Applied Finance, 18(1), pp. 157-172. doi: http://dx.doi. org/10.2139/ssrn.1028206.

Gómez-Ríos, M.C. y D. Juárez-Luna (2018), “Costo de generación eléctrica incorporando externalidades ambientales: Mezcla óptima de tecnologías de carga base", Munich Personal RePEc Archive. <https://mpra.ub.unimuenchen.de/89717/>.

Gantz, D. (2019), "The U.S.-Mexico Trade Relationship under AMLO: Challenges and Opportunities", Arizona Legal Studies Discussion Paper núm. 19-06. EE.UU.

Kimura, S., J. Antón y C. Lethi (2010), "Farm Level Analysis of Risk and Risk Management Strategies and Policies: Cross Country Analysis", OECD Food, Agriculture and Fisheries, Working Papers. <https://doi. org/10.1787/5kmd6b5rl5kd-en>.

León-Herrera, A., M. Martínez-Damián y L. Garza-Bueno (2015), “Comparación de los enfoques media-varianza y media-semivarianza para elegir un portafolio agrícola", Revista Chapingo, serie horticultura (1), pp. 71-80.

Lintner, J. (1965), "The valuation of risk assets on the selection of risky investments in stock portfolios and capital budgets", Review of Economics and Statistics 47(1) pp. 13-37.

Markowitz, H. (1959), Portfolio selection: efficient diversification of investments, vol. 16, EE.UU., Yale University Press.

Merton, R. (1972), "Analytic derivation of the efficient portfolio frontier", Journal of Financial and Quantitative Analysis, pp. 1851-1872.

Mukherjee, S. (2010), "Crop diversification and risk: an empirical analysis of Indian states", Munich Personal RePEc Archive, paper no. 35947. $<$ https://mpra.ub.uni-muenchen.de/35947/>.

Paut, R., R. Sabatier y M. Tchamitchian (2019), "Reducing risks through crop diversification: an apliacation of portfolio theory to diversified horticultural systems", Agricultural Systems, 168, pp. 123-130.

Sharpe, W. (1970), Portfolio theory and capital markets, Inglaterra, McGrawHill College / Sharpe.

Siacon (Sistema de Información Agroalimentaria de Consulta) (2018), "Sistema Estatal de Información para el Desarrollo Rural Sustentable", Secretaría de Agricultura y Desarrollo Rural, Gobierno de México. <https://www. gob.mx/siap/documentos/siacon-ng-161430>.

Vyas, S. (1996), "Diversification in Agriculture: Concept, Rationale and Approaches", Indian Journal of Agricultural Economics, 51(4), pp. 636-643. 